\title{
Poor Vascular Access
}

National Cancer Institute

\section{Source}

National Cancer Institute. Poor Vascular Access. NCI Thesaurus. Code C8281.

A situation describing a high degree of difficultly as it relates to intravenous and/or intraarterial access of an individual's vascular system. 